\title{
A randomized trial of 7-day doripenem versus 10-day imipenem-cilastatin for ventilator- associated pneumonia
}

Marin H Kollef ${ }^{*}$, Jean Chastre ${ }^{2}$, Marc Clavel ${ }^{3}$, Marcos I Restrepo ${ }^{4}$, Bart Michiels ${ }^{5}$, Koné Kaniga ${ }^{6}$, Iolanda Cirillo ${ }^{6}$, Holly Kimko ${ }^{6}$ and Rebecca Redman ${ }^{6}$

\begin{abstract}
Introduction: The aim of this study was to compare a 7-day course of doripenem to a 10-day course of imipenem-cilastatin for ventilator-associated pneumonia (VAP) due to Gram-negative bacteria.

Methods: This was a prospective, double-blinded, randomized trial comparing a fixed 7-day course of doripenem one gram as a four-hour infusion every eight hours with a fixed 10-day course of imipenem-cilastatin one gram as a one-hour infusion every eight hours (April 2008 through June 2011).

Results: The study was stopped prematurely at the recommendation of the Independent Data Monitoring Committee that was blinded to treatment arm assignment and performed a scheduled review of data which showed signals that were close to the pre-specified stopping limits. The final analyses included 274 randomized patients. The clinical cure rate at the end of therapy (EOT) in the microbiological intent-to-treat (MITT) population was numerically lower for patients in the doripenem arm compared to the imipenem-cilastatin arm (45.6\% versus $56.8 \% ; 95 \% \mathrm{Cl},-26.3 \%$ to $3.8 \%$ ). Similarly, the clinical cure rate at EOT was numerically lower for patients with Pseudomonas aeruginosa VAP, the most common Gram-negative pathogen, in the doripenem arm compared to the imipenem-cilastatin arm (41.2\% versus $60.0 \% ; 95 \% \mathrm{Cl},-57.2$ to 19.5$)$. All cause 28 -day mortality in the MITT group was numerically greater for patients in the doripenem arm compared to the imipenem-cilastatin arm (21.5\% versus 14.8\%; $95 \% \mathrm{Cl},-5.0$ to 18.5 ) and for patients with P. aeruginosa VAP (35.3\% versus $0.0 \% ; 95 \% \mathrm{Cl}, 12.6$ to 58.0$)$.

Conclusions: Among patients with microbiologically confirmed late-onset VAP, a fixed 7-day course of doripenem was found to have non-significant higher rates of clinical failure and mortality compared to a fixed 10-day course of imipenem-cilastatin. Consideration should be given to treating patients with VAP for more than seven days to optimize clinical outcome.
\end{abstract}

Trial Registration: ClinicalTrials.gov: NCT00589693

\section{Introduction}

Ventilator-associated pneumonia (VAP) is the most common infection identified in critically ill patients, often due to high risk pathogens, such as Pseudomonas aeruginosa and Acinetobacter baummannii, and accounts for most of the antibiotic utilization within intensive care units (ICUs) $[1,2]$. Several guidelines have been published giving

\footnotetext{
* Correspondence: mkollef@dom.wustl.edu

'Division of Pulmonary and Critical Care Medicine, Washington University School of Medicine, St. Louis, 660 South Euclid Avenue, Campus Box 8052 St. Louis, MO 63110, USA

Full list of author information is available at the end of the article
}

recommendations for the treatment of VAP, including the total duration of therapy [3,4]. Unfortunately, the evidence supporting an optimal duration of antibiotic therapy for VAP is limited and primarily based on the results of a single randomized trial [5]. A recent meta-analysis found that for patients with nosocomial pneumonia not due to nonlactose fermenting Gram-negative bacteria (NLFGNB), a short fixed-course (7 or 8 days) of antibiotic therapy may be more appropriate than a prolonged course (10 to 15 days) in terms of reducing the subsequent emergence of antibiotic-resistant pathogens [6]. However, the concern
() Biomed Central

(c) 2012 Kollef et al.; licensee BioMed Central Ltd. This is an open access article distributed under the terms of the Creative Commons Attribution License (http://creativecommons.org/licenses/by/2.0), which permits unrestricted use, distribution, and reproduction in any medium, provided the original work is properly cited. 
with using short durations of antibiotic therapy is treatment failure and potentially adverse outcomes.

Carbapenems are bactericidal against Gram-negative pathogens that commonly cause VAP, including $P$. aeruginosa, A. baumannii and extended-spectrum beta-lactamase (ESBL) producing enteric bacteria, and are, therefore, recommended for initial empiric therapy for VAP in patients with late-onset disease or individuals with risk factors for infection with multidrug-resistant (MDR) pathogens $[3,4,7]$. Doripenem $500 \mathrm{mg}$ was shown to be non-inferior to comparator agents in two previous randomized controlled studies in patients with hospital-acquired pneumonia, including VAP, when administered for 7 to 14 days, with the length of therapy guided by the patient's condition and at the discretion of the treating physicians $[8,9]$. In addition, pharmacokinetic/pharmacodynamic (PK/PD) modeling from data from other studies demonstrated that one gram doses infused over four hours could target pathogens with higher minimum inhibitory concentrations (MICs) and provide a more sustained duration of free drug concentrations above the MIC of most Gramnegative pathogens causing VAP (especially $P$. aeruginosa and Acinetobacter spp.) than the $500 \mathrm{mg}$ dose $[10,11]$. Therefore, we performed an investigation to compare the administration of a higher $1 \mathrm{~g}$ dose of doripenem for a fixed 7-day course to a fixed10-day course of imipenemcilastatin for the treatment of late-onset VAP. The rationale for the use of a 7-day course of doripenem was guided by data from the prior doripenem nosocomial pneumonia registration trials and a previous study demonstrating similar outcomes in patients with VAP treated with 8 and 15 days of antibiotic therapy [5,8,9].

Doripenem is not approved for treatment of nosocomial pneumonia, including VAP, in the United States (US) but is approved for use in adults with these infections in the European Union and other countries outside of the US.

\section{Materials and methods \\ Study design overview}

A randomized, double-blind, multicenter study was performed comparing the efficacy and safety of a fixed 7-day regimen of doripenem to a fixed 10-day regimen of imipenem-cilastatin in patients with late-onset VAP, with patients enrolled between 1 April 2008 and 17 May 2011. Ventilated patients were stratified at the time of randomization based on age ( $\leq 65$ years or $>65$ years), degree of lung injury as measured by the ratio of the partial pressure of arterial oxygen/fraction of inspired oxygen $(\mathrm{PaO} 2 / \mathrm{FiO} 2$ of $\leq 250$ or $>250$ ), and geographic region (Western Europe, North America, Australia; Central and South America; or Eastern Europe and Asia). The institutional review board at each site (see Acknowledgements) approved the proto$\mathrm{col}$, and all patients or their authorized representatives provided written informed consent (NCT00589693). (See Additional file 1 for complete Methods section).

\section{Randomization and treatment regimens}

In this double-blinded study patients were randomized (1:1) to receive either a fixed 7-day course of doripenem one gram as a four-hour infusion every eight hours or a fixed 10-day course of imipenem-cilastatin one gram as a one-hour infusion every eight hours. Treatment was randomized with use of a central interactive phone system. Randomization was not stratified by study site. Patients randomized to doripenem treatment received in parallel 7 days of active therapy and 10 days of placebo. Patients randomized to imipenem-cilastatin treatment received in parallel 10 days of active therapy and 7 days of placebo. All patients received active study drug and placebo infusions on Days 1 through 7. Patients randomized to imipenemcilastatin continued to receive active study drug on Days 8,9 and 10 and patients randomized to doripenem received placebo. A switch to oral antibacterial therapy was not allowed. Adjunctive therapy was allowed at the discretion of the treating physician with vancomycin ( 1 gram every 12 hours) or linezolid (600 mg every 12 hours) directed at methicillin-resistant Staphylococcus aureus (MRSA) and amikacin ( $15 \mathrm{mg} / \mathrm{kg}$ once daily) for patients at risk for infection with a carbapenem-resistant Gram-negative pathogen.

\section{Outcomes and follow-up}

The intent-to-treat (ITT) population was defined as all patients who received at least one dose of the study drug. The microbiological ITT (MITT) population was the subset of the ITT population who had at least one Gramnegative pathogen identified on bronchoalveolar lavage (BAL) or mini-BAL at a density $\geq 10^{4} \mathrm{CFU} / \mathrm{mL}$ with an imipenem MIC $\leq 8 \mu \mathrm{g} / \mathrm{mL}$. Patients were included in the MITT population if they had a second pathogen isolated from BAL/mini-BAL at a density $\geq 10^{4} \mathrm{CFU} / \mathrm{mL}$ with an imipenem MIC $>8 \mu \mathrm{g} / \mathrm{mL}$. This was allowed to optimize enrollment of patients with eligible Gram-negative pathogens and to allow inclusion of co-infection with MRSA. However, patients who only grew pneumonia pathogens with imipenem MICs $>8 \mu \mathrm{g} / \mathrm{mL}$, such as MRSA or Stenotrophomonas maltophilia, were excluded from the MITT population.

Clinical assessments were performed at baseline and at the end of therapy (EOT), defined as Day 10 for both groups, or within 24 hours after the last dose of blinded study drug therapy if discontinued early. Laboratory assessments were performed at baseline, Day 7 and EOT. Follow-up assessments were conducted 7 to 14 days and 28 to 35 days after EOT. The primary endpoint of this study was clinical cure at EOT (Day 10) in the MITT 
population. Secondary endpoints included 28-day allcause mortality in the MITT populations and clinical cure in the subgroup having $P$. aeruginosa identified as a qualifying pathogen.

Clinical cure was defined as improvement or lack of progression of baseline radiographic findings at EOT and resolution of signs and symptoms of pneumonia at follow-up. Failure was defined as persistence or progression of signs and symptoms or progression of radiological signs of pneumonia at EOT; termination of study medications due to "lack of efficacy"; administration of any systemically absorbed or aerosolized antibiotic for any reason; death from any cause; an indeterminate response; or relapsed infection at follow-up after termination of study medications. Adverse events (AEs) including mortality, vital signs and laboratory parameters were also evaluated.

\section{Statistical analysis}

The initial sample size calculation was based on assumptions from a previous Phase 3 doripenem pneumonia study conducted in patients with VAP [9). Assuming a clinical cure rate of $60 \%$ in both treatment arms and using a non-inferiority margin of $15 \%$ and a one-tailed $2.5 \%$ significance level, a sample size of 168 per treatment arm would have a power of $80 \%$ to establish non-inferiority. If one further assumed that only $70 \%$ of the patients would qualify for inclusion in the MITT analysis set, then the sample size required would be 240 per treatment arm for a total of 480 patients. Categorical data were expressed as frequency distributions and the difference between groups was assessed by using the normal approximation to the difference between two binomial proportions. All confidence intervals were two-tailed and a $P$-value $<0.05$ represented statistical significance. No correction for multiple comparisons was implemented. The $P$-values in the secondary and subgroup analyses are nominal in nature, and not inferential.

\section{Independent Data Monitoring Committee}

An Independent Data Monitoring Committee (IDMC) was established to evaluate data related to efficacy and safety at predefined time points (see on-line supplement for IDMC statistical monitoring guidelines). At their last meeting, the IDMC reviewed available data from approximately half the total number of patients targeted for enrollment and recommended that the enrollment be terminated because of inferior efficacy and higher mortality in one of the treatment arms. Therefore, the analyses were based on data from the 274 subjects who had been randomized into the study at the time enrollment was terminated. In addition, five sites (three in Guatemala, one in Germany, one in the United States) that enrolled a total of 41 patients were deemed to be non-compliant with good clinical practices
(GCP) prior to database lock and were excluded from the primary analyses of efficacy and safety (Figure 1). However, to assess the robustness of the primary efficacy and safety conclusions, sensitivity analyses were performed by including patients from these five sites. These sensitivity analyses support the primary efficacy and safety conclusions.

\section{Results}

\section{Patient disposition and characteristics}

There were 274 randomized patients prior to stopping the study. In addition to the 41 patients from the GCP noncompliant sites, 7 patients were excluded who never received the study drug (1 patient was excluded for meeting both of these criteria). The ITT group comprised 227 patients (doripenem, $n=115$; imipenem-cilastatin, $n=$ 112 ) and the MITT group comprised 167 patients (doripenem, $n=79$; imipenem-cilastatin, $n=88$ ) (Figure 1). Patient baseline characteristics were generally balanced between treatment groups for the ITT and MITT populations although there were some differences between treatment groups suggesting subjects in the doripenem arm may have more severe illness. The majority of patients were male, white, $\leq 65$ years of age (mean of 54.1 years in the MITT population), had an Acute Physiology and Chronic Health Evaluation (APACHE) II score $>15$, a clinical pulmonary infection score (CPIS) $\geq 6, \mathrm{P}_{\mathrm{a}} \mathrm{O}_{2} / \mathrm{F}_{\mathrm{i}} \mathrm{O}_{2} \leq 250$, a creatinine clearance $>50 \mathrm{ml} / \mathrm{min}$, received $>72$ hours of prior antibiotic therapy, and enrolled from sites in Europe (Tables 1 and 2). The most common reasons patients in the MITT population were admitted to the hospital were for surgery (38.9\%), including neurologic surgery (17.4\%), a neurologic event (20.4\%) and trauma (18.6\%). A qualifying Gram-negative pathogen was isolated from $89.2 \%$ of patients in the MITT population and more than half of these patients had a second pathogen isolated from the baseline BAL/mini-BAL at a density $\geq 10^{4} \mathrm{CFU} / \mathrm{mL}$.

The median duration of study drug therapy (including placebo) was 9.7 days for each treatment arm in the MITT population. The median duration of active study drug therapy (excluding placebo) was 7.0 days in the doripenem arm and 10.0 days in the imipenem-cilastatin arm for the MITT population. Similar numbers of patients received empiric adjunctive therapy with an aminoglycoside or an anti-MRSA drug and less than $10 \%$ in both the ITT and MITT groups continued adjunctive antibiotics beyond 72 hours after a carbapenem-resistant pathogen (defined as imipenem MIC $>8 \mu \mathrm{g} / \mathrm{mL}$ ) was isolated (Tables 1 and 2).

\section{Clinical and microbiologic response}

The clinical cure rate at the EOT visit in patients in the MITT group randomized to doripenem was lower than the clinical cure rate in patients randomized to imipenem-cilastatin $(45.6 \%$ versus $56.8 \%$; $95 \% \mathrm{CI},-26.3 \%$ to 


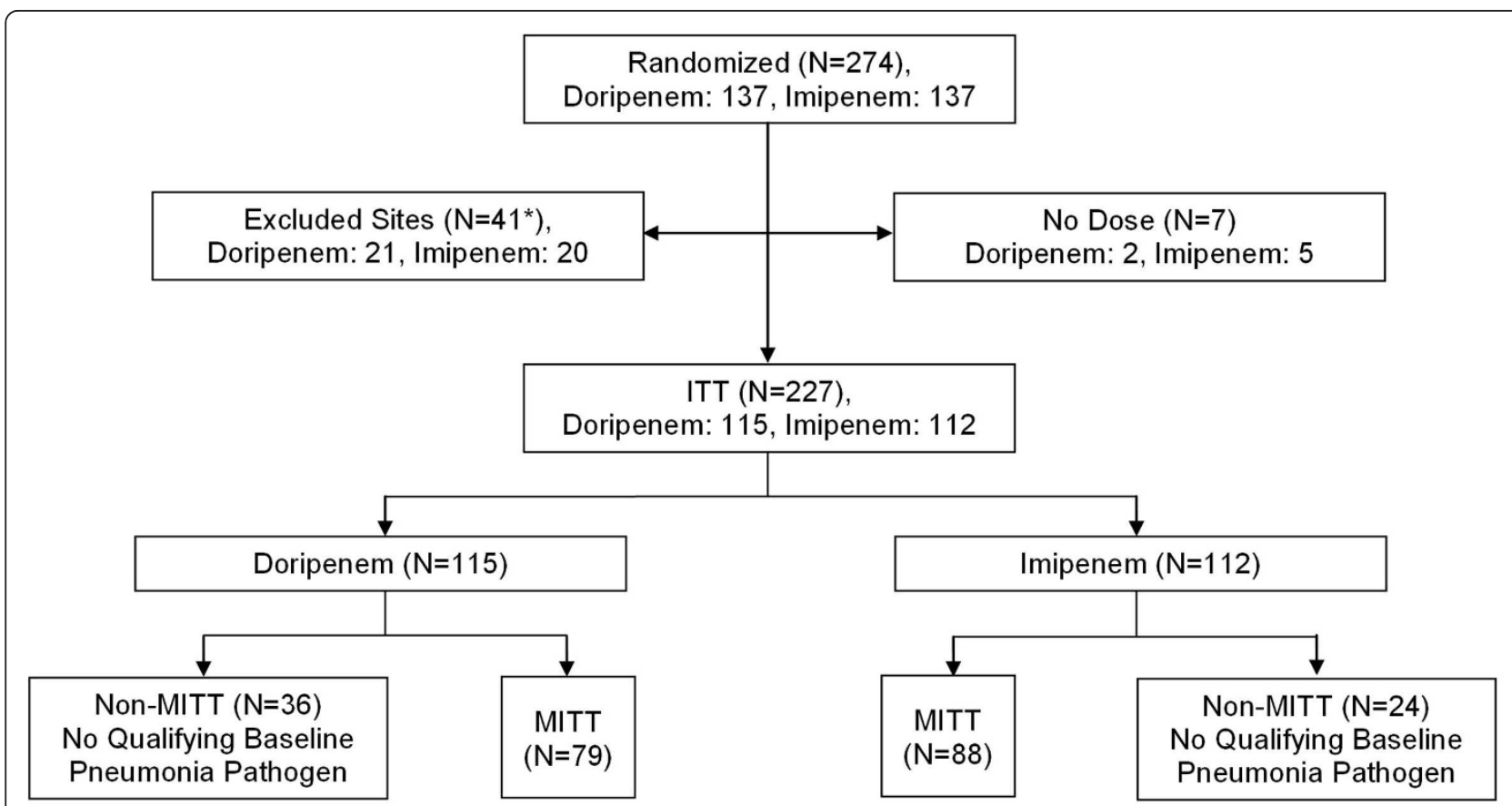

Figure 1 Patients enrolled and analyzed. ITT, intention-to-treat; MITT, Microbiological intention-to-treat. *Prior to study termination the Marketing Authorization Holder for the study identified five study sites (three in Guatemala, one in Germany, one in the United States), following independent internal reviews and re-monitoring by a contract research organization (CRO), that were found not to have adhered to the study protocols, or the study logs could not verify protocol adherence, and thus their data were excluded from the primary analyses.

3.8\%). Thus, non-inferiority of a fixed 7-day treatment regimen with doripenem compared to a fixed 10-day treatment regimen of imipenem-cilastatin was not demonstrated at the 15\% margin. Response differences of $10 \%$ to $15 \%$ favoring imipenem-cilastatin remained present in most subgroups (Figure 2), especially among male patients and those with supra-normal creatinine clearance. However, the larger differences in cure rates in patients with supra-normal creatinine clearance appear to be driven by the unusually high cure rates among subjects in the imipenem-cilastatin arm with creatinine clearance $\geq 150 \mathrm{ml} / \mathrm{min}(71.4 \%)$ compared to cure rates among those with creatinine clearance $\geq 80$ to $<150 \mathrm{ml} / \mathrm{min}(51.4 \%)$ and $>50$ to $<80 \mathrm{ml} / \mathrm{min}(50.0 \%)$.

The mean CPIS values for MITT patients in both treatment arms for study Days 1 through 11 is shown in Figure 3. CPIS scores were similar for patients in the doripenem arm and the imipenem-cilastatin arm for the first eight days of the study. However, the CPIS scores separated after Day 8 with the doripenem arm scores remaining stable while the imipenem-cilastatin arm scores continued to decrease.

The distribution of qualifying Gram-negative pathogens that were pre-defined as being of specific interest $(P$. aeruginosa, Acinetobacter spp., and Enterobacteriaceae) is shown in Table 3. A larger proportion of patients in the doripenem arm than the imipenem-cilastatin arm had pneumonia due to $P$. aeruginosa (21.5\% versus $11.4 \%)$ and Acinetobacter spp. (19.0\% versus $11.4 \%)$. The clinical cure rate for the $P$. aeruginosa subgroup at EOT was numerically lower for subjects in the doripenem arm compared to the imipenem-cilastatin arm $(41.2 \%(7 / 17)$ versus $60.0 \%$ (6/10); $95 \%$ CI, $-57.2 \%$ to $19.5 \%)$. Cure rates were also lower for patients in the doripenem arm infected with Acinetobacter spp. (40.0\% (6/15) versus 50.0\% (5/10); 95\% CI: $-49.7 \%$ to $29.7 \%)$ and Enterobacteriaceae $(53.5 \%$ (23/43) versus $59.2 \%$ (29/49); $95 \%$ CI: $-26.0 \%$ to $14.6 \%$ ). Table 4 shows that the baseline characteristics for the $P$. aeruginosa subgroup were similar between treatment arms.

The number of patients with pathogens at each MIC was too small to draw definitive conclusions regarding clinical cure rate by infecting pathogen MIC; however, for the NLFGNB $P$. aeruginosa and A. baumannii, cure rates and mortality for patients in either treatment arm did not appear to increase with increasing MIC of the study drug received suggesting conditions other than MIC played a role in outcome (see Additional file 1, Table S1).

\section{Safety}

In the ITT population, the most frequently reported adverse events in both treatment groups were anemia (21.7\% doripenem, $22.3 \%$ imipenem-cilastatin); urinary tract infection (13.0\% doripenem, 14.3\% imipenemcilastatin); decubitus ulcer (12.2\% doripenem, $9.8 \%$ 


\begin{tabular}{|c|c|c|c|}
\hline & $\begin{array}{l}\text { Doripenem } \\
(N=115)\end{array}$ & $\begin{array}{l}\text { Imipenem } \\
(N=112)\end{array}$ & $\begin{array}{l}\text { Total } \\
(N=227)\end{array}$ \\
\hline \multicolumn{4}{|l|}{ Sex, n (\%) } \\
\hline N & 115 & 112 & 227 \\
\hline Male & $72(62.6)$ & $75(67.0)$ & $147(64.8)$ \\
\hline Female & $43(37.4)$ & $37(33.0)$ & $80(35.2)$ \\
\hline \multicolumn{4}{|l|}{ Weight (kg) } \\
\hline N & 115 & 112 & 227 \\
\hline Mean (SD) & $75.6(16.95)$ & $79.8(19.07)$ & $77.7(18.11)$ \\
\hline Median & 74.0 & 77.5 & 75.0 \\
\hline Range & $(45 ; 150)$ & $(47 ; 170)$ & $(45 ; 170)$ \\
\hline \multicolumn{4}{|l|}{ Height $(\mathrm{cm})$} \\
\hline N & 114 & 110 & 224 \\
\hline Mean (SD) & $169.5(10.66)$ & $171.3(9.20)$ & $170.3(9.99)$ \\
\hline Median & 170.0 & 170.0 & 170.0 \\
\hline Range & $(140 ; 193)$ & $(150 ; 200)$ & $(140 ; 200)$ \\
\hline \multicolumn{4}{|l|}{ Age (Years) } \\
\hline N & 115 & 112 & 227 \\
\hline Mean (SD) & $57.5(16.53)$ & $54.6(18.46)$ & $56.1(17.53)$ \\
\hline Median & 58.0 & 58.0 & 58.0 \\
\hline Range & $(19 ; 89)$ & $(18 ; 88)$ & $(18 ; 89)$ \\
\hline \multicolumn{4}{|l|}{ Race, n (\%) } \\
\hline N & 115 & 112 & 227 \\
\hline White & $96(83.5)$ & 97 (86.6) & $193(85.0)$ \\
\hline Black or African American & $6(5.2)$ & $6(5.4)$ & $12(5.3)$ \\
\hline Asian & $4(3.5)$ & $1(0.9)$ & $5(2.2)$ \\
\hline Other & $9(7.8)$ & $8(7.1)$ & $17(7.5)$ \\
\hline \multicolumn{4}{|l|}{ Region, n (\%) } \\
\hline N & 115 & 112 & 227 \\
\hline Central and South America & $24(20.9)$ & $24(21.4)$ & $48(21.1)$ \\
\hline Eastern Europe and Asia & $34(29.6)$ & $33(29.5)$ & $67(29.5)$ \\
\hline Western Europe, North America, Australia & $57(49.6)$ & $55(49.1)$ & $112(49.3)$ \\
\hline \multicolumn{4}{|l|}{ APACHE II score, n (\%) } \\
\hline N & 115 & 112 & 227 \\
\hline$\leq 15$ & $48(41.7)$ & $49(43.8)$ & $97(42.7)$ \\
\hline 16 to 19 & $30(26.1)$ & $34(30.4)$ & $64(28.2)$ \\
\hline$\geq 20$ & $37(32.2)$ & $29(25.9)$ & $66(29.1)$ \\
\hline \multicolumn{4}{|l|}{ CPIS, n (\%) } \\
\hline $\mathrm{N}$ & 115 & 112 & 227 \\
\hline Missing & $3(2.6)$ & $2(1.8)$ & $5(2.2)$ \\
\hline$<6$ & $8(7.0)$ & $5(4.5)$ & $13(5.7)$ \\
\hline 6 to 7 & $64(55.7)$ & $64(57.1)$ & $128(56.4)$ \\
\hline 8 to 9 & $30(26.1)$ & $34(30.4)$ & $64(28.2)$ \\
\hline$>9$ & $10(8.7)$ & $7(6.3)$ & $17(7.5)$ \\
\hline \multicolumn{4}{|l|}{ SOFA Score } \\
\hline N & 57 & 58 & 115 \\
\hline Mean (SD) & $6.0(2.70)$ & $5.5(2.39)$ & $5.8(2.55)$ \\
\hline Median & 6.0 & 5.0 & 5.0 \\
\hline Range & $(0 ; 14)$ & $(2 ; 12)$ & $(0 ; 14)$ \\
\hline \multicolumn{4}{|l|}{ Charlson Comorbidity Index } \\
\hline N & 114 & 112 & 226 \\
\hline Mean (SD) & $3.0(2.72)$ & $2.8(2.45)$ & $2.9(2.59)$ \\
\hline
\end{tabular}


Table 1 Baseline demographics - ITT analysis set (Continued)

\begin{tabular}{|c|c|c|c|}
\hline Median & 3.0 & 3.0 & 3.0 \\
\hline Range & $(0 ; 14)$ & $(0 ; 9)$ & $(0 ; 14)$ \\
\hline \multicolumn{4}{|l|}{$\mathrm{PaO}_{2} / \mathrm{FiO}_{2}, \mathrm{n}(\%)$} \\
\hline N & 115 & 112 & 227 \\
\hline$\leq 250$ & $67(58.3)$ & $61(54.5)$ & $128(56.4)$ \\
\hline$>250$ & $48(41.7)$ & $51(45.5)$ & 99 (43.6) \\
\hline \multicolumn{4}{|l|}{ Bacteremia, n (\%) } \\
\hline N & 115 & 112 & 227 \\
\hline No & $109(94.8)$ & $107(95.5)$ & $216(95.2)$ \\
\hline Yes & $6(5.2)$ & $5(4.5)$ & $11(4.8)$ \\
\hline \multicolumn{4}{|l|}{ Creatinine clearance, n (\%) } \\
\hline N & 115 & 112 & 227 \\
\hline Supra normal ( $\geq 150 \mathrm{ml} / \mathrm{min})$ & $23(20.0)$ & $34(30.4)$ & $57(25.1)$ \\
\hline Normal ( $\geq 80$ to $<150 \mathrm{ml} / \mathrm{min}$ ) & $46(40.0)$ & $44(39.3)$ & $90(39.6)$ \\
\hline Mild renal failure ( $>50$ to $<80 \mathrm{ml} / \mathrm{min}$ ) & $33(28.7)$ & $26(23.2)$ & $59(26.0)$ \\
\hline Moderate renal failure (>30 to $\leq 50 \mathrm{ml} / \mathrm{min}$ ) & $7(6.1)$ & $4(3.6)$ & $11(4.8)$ \\
\hline Severe renal failure ( $\leq 30 \mathrm{ml} / \mathrm{min})$ & $6(5.2)$ & $4(3.6)$ & $10(4.4)$ \\
\hline \multicolumn{4}{|l|}{ Failed antibiotic treatment for VAP, $\mathrm{n}(\%)$} \\
\hline $\mathrm{N}$ & 69 & 78 & 147 \\
\hline No & $59(85.5)$ & $67(85.9)$ & $126(85.7)$ \\
\hline Yes & $10(14.5)$ & $11(14.1)$ & $21(14.3)$ \\
\hline \multicolumn{4}{|l|}{ Prior antibacterial therapy usage (hours), $\mathrm{n}(\%)$} \\
\hline $\mathrm{N}$ & 115 & 112 & 227 \\
\hline$<24$ & $28(24.3)$ & $35(31.3)$ & $63(27.8)$ \\
\hline$\geq 24$ to $<48$ & $10(8.7)$ & $10(8.9)$ & $20(8.8)$ \\
\hline$\geq 48$ to $\leq 72$ & $9(7.8)$ & $5(4.5)$ & $14(6.2)$ \\
\hline$>72$ & $68(59.1)$ & $62(55.4)$ & $130(57.3)$ \\
\hline \multicolumn{4}{|l|}{ Adjunctive therapy, $\mathrm{n}(\%)$} \\
\hline N & 115 & 112 & 227 \\
\hline No & $75(65.2)$ & $79(70.5)$ & $154(67.8)$ \\
\hline \multicolumn{4}{|l|}{ Yes } \\
\hline$\leq 72 \mathrm{Hrs}$ & $32(27.8)$ & $25(22.3)$ & $57(25.1)$ \\
\hline$>72 \mathrm{Hrs}$ & $8(7.0)$ & $8(7.1)$ & $16(7.0)$ \\
\hline \multicolumn{4}{|l|}{ Adjunctive aminoglycoside, $\mathrm{n}(\%)$} \\
\hline N & 40 & 33 & 73 \\
\hline No & $19(47.5)$ & $20(60.6)$ & $39(53.4)$ \\
\hline \multicolumn{4}{|l|}{ Yes } \\
\hline$\leq 72 \mathrm{Hrs}$ & $20(50.0)$ & $12(36.4)$ & $32(43.8)$ \\
\hline$>72 \mathrm{Hrs}$ & $1(2.5)$ & $1(3.0)$ & $2(2.7)$ \\
\hline \multicolumn{4}{|l|}{ Adjunctive vancomycin/linezolid, n (\%) } \\
\hline N & 40 & 33 & 73 \\
\hline No & $14(35.0)$ & $9(27.3)$ & $23(31.5)$ \\
\hline \multicolumn{4}{|l|}{ Yes } \\
\hline$\leq 72$ hrs & $20(50.0)$ & $18(54.5)$ & $38(52.1)$ \\
\hline$>72$ hrs & $6(15.0)$ & $6(18.2)$ & $12(16.4)$ \\
\hline
\end{tabular}

APACHE, Acute Physiology and Chronic Health Evaluation; CPIS, Clinical Pulmonary Infection Score; ITT, intention-to-treat; SOFA, Sequential Organ Failure Assessment

imipenem-cilastatin); hypokalemia (10.4\% doripenem, $10.7 \%$ imipenem-cilastatin); diarrhea (9.6\% doripenem, $11.6 \%$ imipenem-cilastatin); and hypotension (9.6\% doripenem, $8.9 \%$ imipenem-cilastatin). Clinically important adverse events (all-causality) are shown in Additional file 1. Laboratory results were also comparable between the study arms (see Additional file 1, Table S2). 


\begin{tabular}{|c|c|c|c|}
\hline & $\begin{array}{c}\text { Doripenem } \\
(N=79)\end{array}$ & $\begin{array}{c}\text { Imipenem } \\
(N=88)\end{array}$ & $\begin{array}{c}\text { Total } \\
(N=167)\end{array}$ \\
\hline \multicolumn{4}{|l|}{ Sex, n (\%) } \\
\hline N & 79 & 88 & 167 \\
\hline Male & $48(60.8)$ & $61(69.3)$ & $109(65.3)$ \\
\hline Female & $31(39.2)$ & $27(30.7)$ & $58(34.7)$ \\
\hline \multicolumn{4}{|l|}{ Weight (kg) } \\
\hline N & 79 & 88 & 167 \\
\hline Mean (SD) & $75.5(17.85)$ & $78.7(15.66)$ & $77.2(16.75)$ \\
\hline Median & 75.0 & 78.0 & 76.0 \\
\hline Range & $(45 ; 150)$ & $(47 ; 143)$ & $(45 ; 150)$ \\
\hline \multicolumn{4}{|l|}{ Height $(\mathrm{cm})$} \\
\hline N & 79 & 87 & 166 \\
\hline Mean (SD) & $170.8(9.76)$ & $171.7(8.73)$ & $171.3(9.22)$ \\
\hline Median & 170.0 & 170.0 & 170.0 \\
\hline Range & $(148 ; 193)$ & $(150 ; 190)$ & $(148 ; 193)$ \\
\hline \multicolumn{4}{|l|}{ Age (years) } \\
\hline $\mathrm{N}$ & 79 & 88 & 167 \\
\hline Mean (SD) & $54.9(16.10)$ & $53.4(18.94)$ & $54.1(17.62)$ \\
\hline Median & 56.0 & 57.0 & 57.0 \\
\hline Range & $(19 ; 89)$ & $(18 ; 88)$ & $(18 ; 89)$ \\
\hline \multicolumn{4}{|l|}{ Race, n (\%) } \\
\hline $\mathrm{N}$ & 79 & 88 & 167 \\
\hline White & $65(82.3)$ & $75(85.2)$ & $140(83.8)$ \\
\hline Black or African American & $5(6.3)$ & $6(6.8)$ & $11(6.6)$ \\
\hline Asian & $1(1.3)$ & $1(1.1)$ & $2(1.2)$ \\
\hline Other & $8(10.1)$ & $6(6.8)$ & $14(8.4)$ \\
\hline \multicolumn{4}{|l|}{ Region, n (\%) } \\
\hline N & 79 & 88 & 167 \\
\hline Central and South America & $20(25.3)$ & $19(21.6)$ & $39(23.4)$ \\
\hline Eastern Europe and Asia & $24(30.4)$ & $27(30.7)$ & $51(30.5)$ \\
\hline Western Europe, North America, Australia & $35(44.3)$ & $42(47.7)$ & $77(46.1)$ \\
\hline \multicolumn{4}{|l|}{ APACHE II score group, $n$ (\%) } \\
\hline N & 79 & 88 & 167 \\
\hline$\leq 15$ & $34(43.0)$ & $42(47.7)$ & $76(45.5)$ \\
\hline 16 to 19 & $24(30.4)$ & $21(23.9)$ & $45(26.9)$ \\
\hline$\geq 20$ & $21(26.6)$ & $25(28.4)$ & $46(27.5)$ \\
\hline \multicolumn{4}{|l|}{ CPIS, n (\%) } \\
\hline N & 79 & 88 & 167 \\
\hline Missing & $2(2.5)$ & $1(1.1)$ & $3(1.8)$ \\
\hline$<6$ & $4(5.1)$ & $2(2.3)$ & $6(3.6)$ \\
\hline 6 to 7 & $43(54.4)$ & $50(56.8)$ & $93(55.7)$ \\
\hline 8 to 9 & $23(29.1)$ & $29(33.0)$ & $52(31.1)$ \\
\hline$>9$ & $7(8.9)$ & $6(6.8)$ & $13(7.8)$ \\
\hline \multicolumn{4}{|l|}{ SOFA score } \\
\hline $\mathrm{N}$ & 41 & 46 & 87 \\
\hline Mean (SD) & $5.7(2.53)$ & $5.2(2.24)$ & $5.4(2.38)$ \\
\hline Median & 6.0 & 5.0 & 5.0 \\
\hline Range & $(0 ; 11)$ & $(2 ; 12)$ & $(0 ; 12)$ \\
\hline \multicolumn{4}{|l|}{ Charlson Comorbidity Index } \\
\hline $\mathrm{N}$ & 79 & 88 & 167 \\
\hline Mean (SD) & $2.4(2.08)$ & $2.8(2.48)$ & $2.6(2.30)$ \\
\hline
\end{tabular}


Table 2 Baseline demographics - MITT analysis set (Continued)

\begin{tabular}{|c|c|c|c|}
\hline Median & 3.0 & 2.0 & 3.0 \\
\hline Range & $(0 ; 9)$ & $(0 ; 9)$ & $(0 ; 9)$ \\
\hline \multicolumn{4}{|l|}{$\mathrm{PaO}_{2} / \mathrm{FiO}_{2}, \mathrm{n}(\%)$} \\
\hline N & 79 & 88 & 167 \\
\hline$\leq 250$ & $50(63.3)$ & $51(58.0)$ & $101(60.5)$ \\
\hline$>250$ & $29(36.7)$ & $37(42.0)$ & $66(39.5)$ \\
\hline \multicolumn{4}{|l|}{ Bacteremia, n (\%) } \\
\hline N & 79 & 88 & 167 \\
\hline No & $73(92.4)$ & $84(95.5)$ & $157(94.0)$ \\
\hline Yes & $6(7.6)$ & $4(4.5)$ & $10(6.0)$ \\
\hline \multicolumn{4}{|l|}{ Creatinine Clearance, n (\%) } \\
\hline N & 79 & 88 & 167 \\
\hline Supra normal ( $\geq 150 \mathrm{ml} / \mathrm{min})$ & $18(22.8)$ & $28(31.8)$ & $46(27.5)$ \\
\hline Normal ( $\geq 80$ to $<150 \mathrm{ml} / \mathrm{min}$ ) & $31(39.2)$ & $37(42.0)$ & $68(40.7)$ \\
\hline Mild renal failure ( $>50$ to $<80 \mathrm{ml} / \mathrm{min}$ ) & $23(29.1)$ & $18(20.5)$ & $41(24.6)$ \\
\hline Moderate renal failure (>30 to $\leq 50 \mathrm{ml} / \mathrm{min}$ ) & $5(6.3)$ & $2(2.3)$ & $7(4.2)$ \\
\hline Severe renal failure ( $\leq 30 \mathrm{ml} / \mathrm{min})$ & $2(2.5)$ & $3(3.4)$ & $5(3.0)$ \\
\hline \multicolumn{4}{|l|}{ Failed antibiotic treatment, $\mathrm{n}(\%)$} \\
\hline N & 55 & 68 & 123 \\
\hline No & $45(81.8)$ & $58(85.3)$ & $103(83.7)$ \\
\hline Yes & $10(18.2)$ & $10(14.7)$ & $20(16.3)$ \\
\hline \multicolumn{4}{|l|}{ Prior antibacterial therapy usage (hours), $n$ (\%) } \\
\hline N & 79 & 88 & 167 \\
\hline$<24$ & $19(24.1)$ & $29(33.0)$ & $48(28.7)$ \\
\hline$\geq 24$ to $<48$ & $8(10.1)$ & $8(9.1)$ & $16(9.6)$ \\
\hline$\geq 48$ to $\leq 72$ & $6(7.6)$ & $3(3.4)$ & $9(5.4)$ \\
\hline$>72$ & $46(58.2)$ & $48(54.5)$ & $94(56.3)$ \\
\hline \multicolumn{4}{|l|}{ Adjunctive therapy, $\mathrm{n}(\%)$} \\
\hline $\mathrm{N}$ & 79 & 88 & 167 \\
\hline No & $49(62.0)$ & $68(77.3)$ & $117(70.1)$ \\
\hline \multicolumn{4}{|l|}{ Yes } \\
\hline$\leq 72 \mathrm{Hrs}$ & $23(29.1)$ & $15(17.0)$ & $38(22.8)$ \\
\hline$>72 \mathrm{Hrs}$ & $7(8.9)$ & $5(5.7)$ & $12(7.2)$ \\
\hline \multicolumn{4}{|l|}{ Adjunctive aminoglycoside, $n(\%)$} \\
\hline N & 30 & 20 & 50 \\
\hline No & $15(50.0)$ & $11(55.0)$ & $26(52.0)$ \\
\hline \multicolumn{4}{|l|}{ Yes } \\
\hline$\leq 72$ hrs & $14(46.7)$ & $8(40.0)$ & $22(44.0)$ \\
\hline$>72$ hrs & $1(3.3)$ & $1(5.0)$ & $2(4.0)$ \\
\hline \multicolumn{4}{|l|}{ Adjunctive vancomycin/linezolid, n (\%) } \\
\hline N & 30 & 20 & 50 \\
\hline No & $9(30.0)$ & $6(30.0)$ & $15(30.0)$ \\
\hline \multicolumn{4}{|l|}{ Yes } \\
\hline$\leq 72 \mathrm{hrs}$ & $16(53.3)$ & $11(55.0)$ & $27(54.0)$ \\
\hline$>72$ hrs & $5(16.7)$ & $3(15.0)$ & $8(16.0)$ \\
\hline
\end{tabular}

APACHE, Acute Physiology and Chronic Health Evaluation; CPIS, Clinical Pulmonary Infection Score; MITT, microbiological intention-to -treat; SOFA, Sequential Organ Failure Assessment

All-cause 28-day mortality in the MITT group was numerically higher for patients in the doripenem arm compared to the imipenem-cilastatin arm (21.5\% (17/79) doripenem, 14.8\% (13/88) imipenem-cilastatin; 95\% CI,
$-5.0 \%$ to $18.5 \%)$ and greater for patients with $P$. aeruginosa VAP (35.3\% (6/17) versus $0.0 \%$ (0/10); 95\% CI, $12.6 \%$ to $58.0 \%)$. Similar trends but smaller mortality differences were demonstrated for the sensitivity 


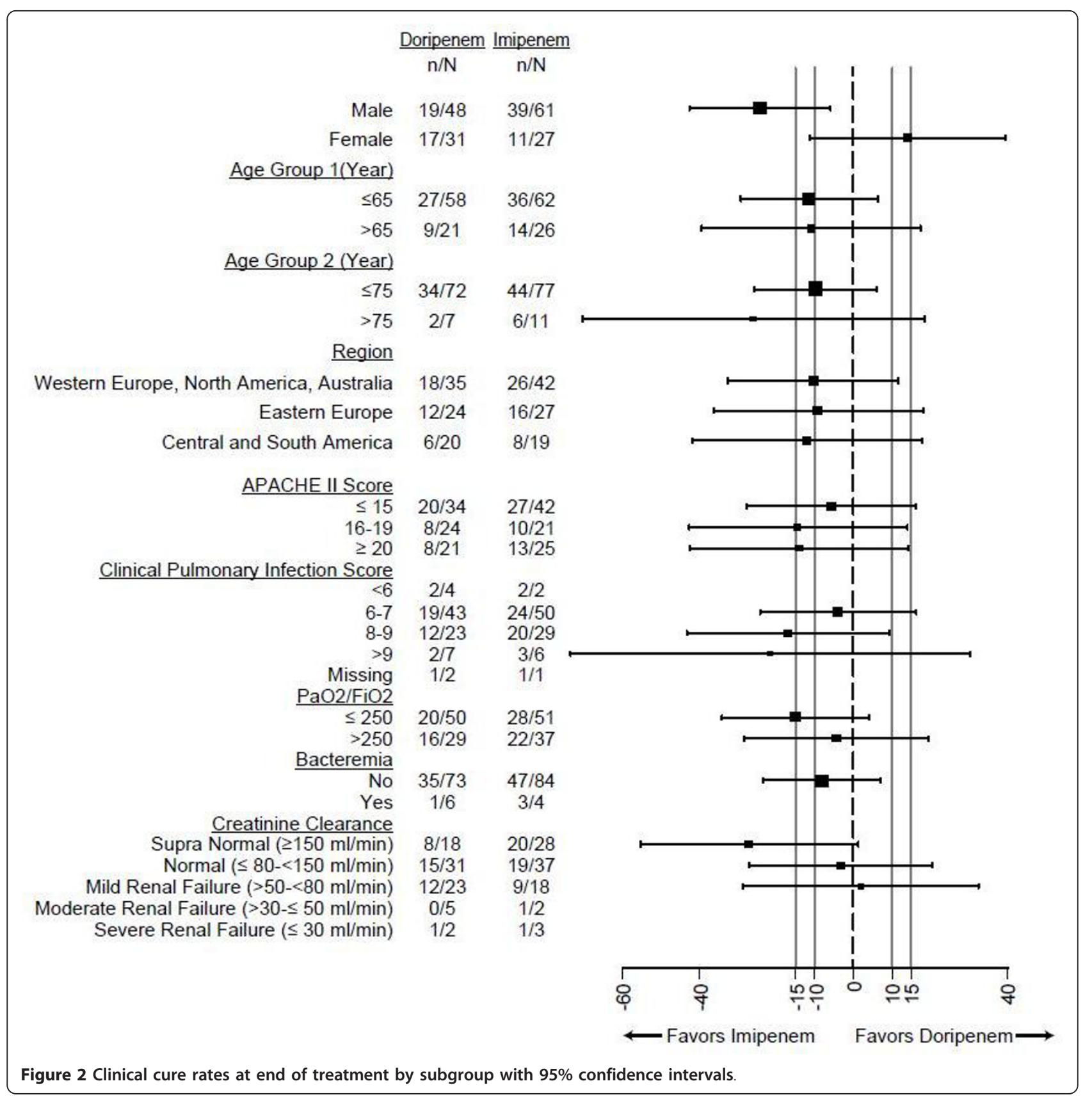

analyses for the MITT group (20.7\% (19/92) doripenem, $16.7 \%$ (17/102) imipenem-cilastatin; 95\% CI, $-7.0 \%$ to $15.0 \%)$. Kaplan-Meier estimates are presented graphically for 28-day all-cause mortality for the ITT and MITT populations (see Additional file 1). Figure 4 demonstrates that the Kaplan-Meier curves for the $P$. aeruginosa subgroup are statistically, significantly different over the treatment arms (nominal $P$-value $=0.040$ ) with an increased separation after completion of study drug administration. Notably, no patient in the imipenem arm with $P$. aeruginosa VAP died. In contrast, mortality rates for patients with Acinetobacter spp. VAP were lower for patients treated with doripenem $(13.3 \%$ (2/15) versus $30.0 \%$ (3/10); $95 \%$ CI: $-49.9 \%$ to $16.5 \%)$.

\section{Pharmacokinetics}

The concentration data collected from 43 subjects treated with doripenem (between study Days 2 and 3) were within the range of historical data in previously studied critically ill patients administered doripenem $1 \mathrm{~g}$ for a four-hour infusion. This data were utilized in a population $\mathrm{PK} / \mathrm{PD}$ analysis along with data from subjects with 


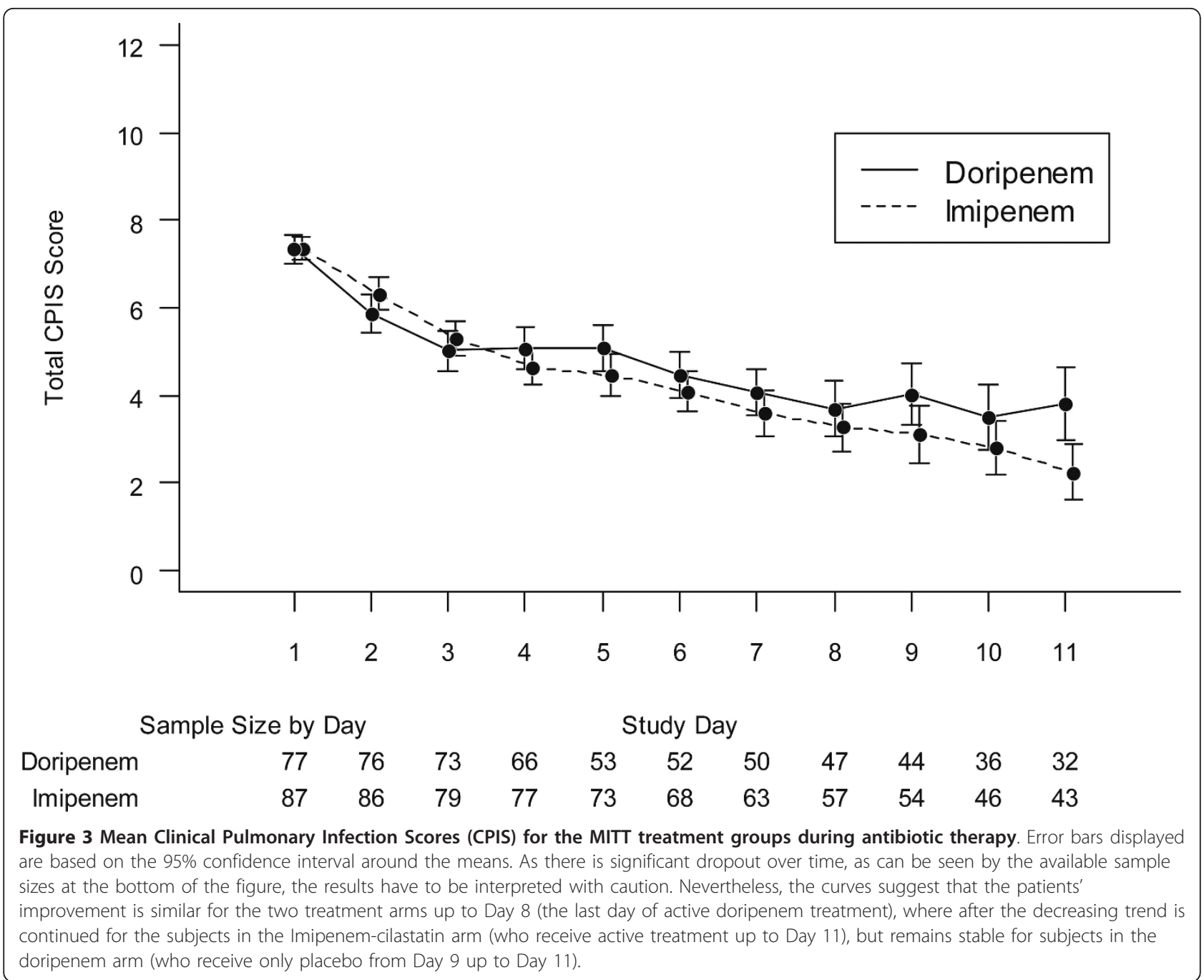

VAP from previously conducted studies (manuscript under preparation). Higher volumes of distribution were observed in this study population, likely attributable to high peripheral fluid volumes and the VAP disease state. Despite this, doripenem levels were maintained at levels sufficient to target pathogens isolated from subjects in this study.

\section{Discussion}

The main reasons to consider the use of shorter courses of antibiotic therapy for VAP are to minimize antibioticrelated complications and to prevent the emergence of antibiotic resistance. However, we demonstrated that among patients with microbiologically confirmed VAP, a fixed 7-day course of doripenem (one gram as a fourhour infusion every eight hours) had non-significant higher rates of clinical failure and mortality compared to a fixed 10-day course of imipenem-cilastatin (one gram as a one-hour infusion every eight hours). Moreover, patients with VAP attributed to $P$. aeruginosa had a statistically greater risk of 28-day all-cause mortality when treated with doripenem compared to imipenem-cilastatin with an increased separation in the survival curves after completion of study drug administration. This occurred despite the use of prolonged infusions of doripenem aimed at optimizing antibiotic concentration target attainment above the MIC of bacterial pathogens during the dosing interval suggesting that the shorter course of doripenem administration played a role in this survival difference [12,13].

Our findings are in contrast to some earlier studies suggesting that shorter courses of antibiotic therapy for VAP are safe and efficacious compared to longer treatment courses. Ibrahim et al. showed that implementation of a clinical guideline for the treatment of VAP was associated with greater administration of appropriate initial antimicrobial treatment [14]. The duration of antimicrobial treatment was also statistically shorter 
Table 3 Distribution of baseline qualifying Gram-negative pathogens

\begin{tabular}{|c|c|c|c|}
\hline & $\begin{array}{c}\text { Doripenem } \\
(N=79) \\
\mathrm{n}(\%)\end{array}$ & $\begin{array}{c}\text { Imipenem } \\
(N=88) \\
\text { n (\%) }\end{array}$ & $\begin{array}{c}\text { Total } \\
(N=167) \\
\mathrm{n}(\%)\end{array}$ \\
\hline Specific Gram-negative Pathogens & $65(82.3)$ & $62(70.5)$ & $127(76.0)$ \\
\hline Pseudomonas aeruginosa & $17(21.5)$ & $10(11.4)$ & $27(16.2)$ \\
\hline Monomicrobial* & $8(10.1)$ & $6(6.8)$ & $14(8.4)$ \\
\hline Polymicrobial** & $9(11.4)$ & $4(4.5)$ & $13(7.8)$ \\
\hline Acinetobacter spp. & $15(19.0)$ & $10(11.4)$ & $25(15.0)$ \\
\hline Monomicrobial* & $3(3.8)$ & $4(4.5)$ & $7(4.2)$ \\
\hline Polymicrobial** & $12(15.2)$ & $6(6.8)$ & $18(10.8)$ \\
\hline Enterobacteriaceae & $43(54.4)$ & $49(55.7)$ & $92(55.1)$ \\
\hline Monomicrobial* & $14(17.7)$ & $14(15.9)$ & $28(16.8)$ \\
\hline Citrobacter koseri & $1(1.3)$ & 0 & $1(0.6)$ \\
\hline Enterobacter aerogenes & 0 & $1(1.1)$ & $1(0.6)$ \\
\hline Enterobacter cloacae & $4(5.1)$ & $3(3.4)$ & $7(4.2)$ \\
\hline Escherichia coli & $1(1.3)$ & $2(2.3)$ & $3(1.8)$ \\
\hline Klebsiella pneumoniae & $5(6.3)$ & $4(4.5)$ & $9(5.4)$ \\
\hline Pantoea agglomerans & $1(1.3)$ & 0 & $1(0.6)$ \\
\hline Proteus mirabilis & $1(1.3)$ & $2(2.3)$ & $3(1.8)$ \\
\hline Serratia marcescens & $1(1.3)$ & $2(2.3)$ & $3(1.8)$ \\
\hline Polymicrobial** & $29(36.7)$ & $35(39.8)$ & $64(38.3)$ \\
\hline Citrobacter freundii & 0 & $3(3.4)$ & $3(1.8)$ \\
\hline Enterobacter aerogenes & 0 & $2(2.3)$ & $2(1.2)$ \\
\hline Enterobacter asburiae & $1(1.3)$ & 0 & $1(0.6)$ \\
\hline Enterobacter cloacae & $4(5.1)$ & $2(2.3)$ & $6(3.6)$ \\
\hline Enterobacter spp. (Not Speciated) & $1(1.3)$ & 0 & $1(0.6)$ \\
\hline Escherichia coli & $5(6.3)$ & $12(13.6)$ & $17(10.2)$ \\
\hline Klebsiella oxytoca & $1(1.3)$ & $1(1.1)$ & $2(1.2)$ \\
\hline Klebsiella pneumoniae & $14(17.7)$ & $16(18.2)$ & $30(18.0)$ \\
\hline Proteus mirabilis & $3(3.8)$ & $3(3.4)$ & $6(3.6)$ \\
\hline Proteus vulgaris & $1(1.3)$ & $1(1.1)$ & $2(1.2)$ \\
\hline Providencia spp. (Not Speciated) & 0 & $1(1.1)$ & $1(0.6)$ \\
\hline Serratia marcescens & $3(3.8)$ & $3(3.4)$ & $6(3.6)$ \\
\hline Pneumonia with MRS & $7(8.9)$ & $4(4.5)$ & $11(6.6)$ \\
\hline Pseudomonas aeruginosa & $2(2.5)$ & $1(1.1)$ & $3(1.8)$ \\
\hline Polymicrobial** & $2(2.5)$ & $1(1.1)$ & $3(1.8)$ \\
\hline Acinetobacter spp. & $2(2.5)$ & $1(1.1)$ & $3(1.8)$ \\
\hline Polymicrobial** & $2(2.5)$ & $1(1.1)$ & $3(1.8)$ \\
\hline Enterobacteriaceae & $4(5.1)$ & $2(2.3)$ & $6(3.6)$ \\
\hline Polymicrobial** & $4(5.1)$ & $2(2.3)$ & $6(3.6)$ \\
\hline
\end{tabular}

*: Monomicrobial pneumonia means a single qualifying pneumonia pathogen was isolated and no other pneumonia pathogen was isolated. **: Polymicrobial pneumonia means at least one qualifying pneumonia pathogen was isolated and at least one other pneumonia pathogen was isolated. MRS, methicillin-resistant Staphylococcus

with use of the guideline $(8.6 \pm 5.1$ days versus $14.8 \pm$ 8.1 days, $P<0.001$ ) and second episodes of VAP occurred statistically less often. In a subsequent study the same group of investigators found that an antibiotic discontinuation policy for clinically suspected VAP, overseen by clinical pharmacists who were part of the ICU team, could also significantly reduce the duration of antibiotic therapy compared to therapy determined by the treating physician teams $(6.0+4.9$ days versus
$8.0 \pm 5.6$ days, $P=0.001)[15]$. Secondary outcomes including relapse of VAP, hospital mortality, and lengths of stay were similar between groups, although the number of infections attributed to NLFGNB was small. Several groups have also employed prediction tools like CPIS and the biomarker procalcitonin to successfully reduce the duration of antimicrobial therapy in patients with VAP without adversely influencing patient outcomes [16-18]. 
Table 4 Baseline clinical characteristics - $P$.

\begin{tabular}{|c|c|c|c|}
\hline & $\begin{array}{l}\text { Doripenem } \\
(N=17)\end{array}$ & $\begin{array}{l}\text { Imipenem } \\
(N=10)\end{array}$ & $\begin{array}{c}\text { Total } \\
(N=27)\end{array}$ \\
\hline \multicolumn{4}{|l|}{ Sex, n (\%) } \\
\hline N & 17 & 10 & 27 \\
\hline Male & $13(76.5)$ & $7(70.0)$ & $20(74.1)$ \\
\hline Female & $4(23.5)$ & $3(30.0)$ & $7(25.9)$ \\
\hline \multicolumn{4}{|l|}{ Weight (kg) } \\
\hline N & 17 & 10 & 27 \\
\hline Mean (SD) & $74.0(17.85)$ & $68.3(12.12)$ & $71.9(15.96)$ \\
\hline Median & 75.0 & 64.0 & 72.7 \\
\hline Range & $(45 ; 110)$ & $(52 ; 90)$ & $(45 ; 110)$ \\
\hline \multicolumn{4}{|l|}{ Height $(\mathrm{cm})$} \\
\hline N & 17 & 10 & 27 \\
\hline Mean (SD) & $172.0(10.11)$ & $170.8(7.74)$ & $171.6(9.17)$ \\
\hline Median & 170.0 & 171.0 & 170.0 \\
\hline Range & $(156 ; 190)$ & $(159 ; 180)$ & $(156 ; 190)$ \\
\hline \multicolumn{4}{|l|}{ Age (Years) } \\
\hline N & 17 & 10 & 27 \\
\hline Mean (SD) & $57.5(15.58)$ & $50.9(20.32)$ & $55.1(17.40)$ \\
\hline Median & 57.0 & 53.5 & 57.0 \\
\hline Range & $(33 ; 89)$ & $(25 ; 77)$ & $(25 ; 89)$ \\
\hline \multicolumn{4}{|l|}{ Race, n (\%) } \\
\hline $\mathrm{N}$ & 17 & 10 & 27 \\
\hline White & $16(94.1)$ & $7(70.0)$ & $23(85.2)$ \\
\hline Black or African American & $1(5.9)$ & $2(20.0)$ & $3(11.1)$ \\
\hline Asian & 0 & $1(10.0)$ & $1(3.7)$ \\
\hline \multicolumn{4}{|l|}{ Region, n (\%) } \\
\hline N & 17 & 10 & 27 \\
\hline Central and South America & $5(29.4)$ & $3(30.0)$ & $8(29.6)$ \\
\hline Eastern Europe and Asia & $5(29.4)$ & $4(40.0)$ & $9(33.3)$ \\
\hline Western Europe, North America, Australia & $7(41.2)$ & $3(30.0)$ & $10(37.0)$ \\
\hline \multicolumn{4}{|l|}{ APACHE II score group, n (\%) } \\
\hline N & 17 & 10 & 27 \\
\hline$\leq 15$ & $6(35.3)$ & $3(30.0)$ & $9(33.3)$ \\
\hline 16 to 19 & $5(29.4)$ & $3(30.0)$ & $8(29.6)$ \\
\hline$\geq 20$ & $6(35.3)$ & $4(40.0)$ & $10(37.0)$ \\
\hline \multicolumn{4}{|l|}{ CPIS, n (\%) } \\
\hline $\mathrm{N}$ & 17 & 10 & 27 \\
\hline$<6$ & 0 & $1(10.0)$ & $1(3.7)$ \\
\hline 6 to 7 & $10(58.8)$ & $8(80.0)$ & $18(66.7)$ \\
\hline 8 to 9 & $5(29.4)$ & $1(10.0)$ & $6(22.2)$ \\
\hline$>9$ & $2(11.8)$ & 0 & $2(7.4)$ \\
\hline \multicolumn{4}{|l|}{ SOFA score } \\
\hline N & 10 & 4 & 14 \\
\hline Mean (SD) & $5.4(3.10)$ & $4.0(1.41)$ & $5.0(2.75)$ \\
\hline Median & 4.0 & 4.5 & 4.0 \\
\hline Range & $(1 ; 10)$ & $(2 ; 5)$ & $(1 ; 10)$ \\
\hline \multicolumn{4}{|l|}{ Charlson Comorbidity Index } \\
\hline $\mathrm{N}$ & 17 & 10 & 27 \\
\hline Mean (SD) & $3.0(2.26)$ & $2.5(1.96)$ & $2.8(2.13)$ \\
\hline Median & 3.0 & 3.0 & 3.0 \\
\hline Range & $(0 ; 9)$ & $(0 ; 5)$ & $(0 ; 9)$ \\
\hline
\end{tabular}


Table 4 Baseline clinical characteristics - P. (Continued)

\begin{tabular}{|c|c|c|c|}
\hline \multicolumn{4}{|l|}{$\mathrm{PaO}_{2} / \mathrm{FiO}_{2}, \mathrm{n}(\%)$} \\
\hline $\mathrm{N}$ & 17 & 10 & 27 \\
\hline$\leq 250$ & $10(58.8)$ & $5(50.0)$ & $15(55.6)$ \\
\hline$>250$ & $7(41.2)$ & $5(50.0)$ & $12(44.4)$ \\
\hline \multicolumn{4}{|l|}{ Bacteremia, n (\%) } \\
\hline $\mathrm{N}$ & 17 & 10 & 27 \\
\hline No & $16(94.1)$ & $10(100)$ & $26(96.3)$ \\
\hline Yes & $1(5.9)$ & 0 & $1(3.7)$ \\
\hline \multicolumn{4}{|l|}{ Creatinine clearance, n (\%) } \\
\hline N & 17 & 10 & 27 \\
\hline Supra normal( $\geq 150$ ml/min) & $5(29.4)$ & $4(40.0)$ & $9(33.3)$ \\
\hline Normal ( $\geq 80$ to $<150 \mathrm{ml} / \mathrm{min}$ ) & $5(29.4)$ & $2(20.0)$ & $7(25.9)$ \\
\hline Mild renal failure (>50 to $<80 \mathrm{ml} / \mathrm{min}$ ) & $4(23.5)$ & $3(30.0)$ & $7(25.9)$ \\
\hline Moderate renal failure (>30 to $\leq 50 \mathrm{ml} / \mathrm{min}$ ) & $2(11.8)$ & $1(10.0)$ & $3(11.1)$ \\
\hline Severe renal failure $(\leq 30 \mathrm{ml} / \mathrm{min})$ & $1(5.9)$ & 0 & $1(3.7)$ \\
\hline \multicolumn{4}{|l|}{ Failed antibiotic treatment, $\mathrm{n}(\%)$} \\
\hline $\mathrm{N}$ & 11 & 8 & 19 \\
\hline No & $8(72.7)$ & $6(75.0)$ & $14(73.7)$ \\
\hline Yes & $3(27.3)$ & $2(25.0)$ & $5(26.3)$ \\
\hline \multicolumn{4}{|l|}{ Prior antibacterial therapy usage (hours), $\mathbf{n}(\%)$} \\
\hline $\mathrm{N}$ & 17 & 10 & 27 \\
\hline$<24$ & $4(23.5)$ & 0 & $4(14.8)$ \\
\hline$\geq 24$ to $<48$ & $2(11.8)$ & $1(10.0)$ & $3(11.1)$ \\
\hline$\geq 48$ to $\leq 72$ & $1(5.9)$ & 0 & $1(3.7)$ \\
\hline$>72$ & $10(58.8)$ & $9(90.0)$ & $19(70.4)$ \\
\hline \multicolumn{4}{|l|}{ Adjunctive therapy, $\mathrm{n}(\%)$} \\
\hline N & 17 & 10 & 27 \\
\hline No & $7(41.2)$ & $6(60.0)$ & $13(48.1)$ \\
\hline \multicolumn{4}{|l|}{ Yes } \\
\hline$\leq 72$ hrs & $8(47.1)$ & $3(30.0)$ & $11(40.7)$ \\
\hline$>72$ hrs & $2(11.8)$ & $1(10.0)$ & $3(11.1)$ \\
\hline \multicolumn{4}{|l|}{ Adjunctive aminoglycoside, n (\%) } \\
\hline $\mathrm{N}$ & 10 & 4 & 14 \\
\hline No & $4(40.0)$ & $2(50.0)$ & $6(42.9)$ \\
\hline \multicolumn{4}{|l|}{ Yes } \\
\hline$\leq 72$ hrs & $5(50.0)$ & $2(50.0)$ & $7(50.0)$ \\
\hline$>72$ hrs & $1(10.0)$ & 0 & $1(7.1)$ \\
\hline \multicolumn{4}{|l|}{ Adjunctive vancomycin/linezolid, n (\%) } \\
\hline $\mathrm{N}$ & 10 & 4 & 14 \\
\hline No & $5(50.0)$ & $1(25.0)$ & $6(42.9)$ \\
\hline \multicolumn{4}{|l|}{ Yes } \\
\hline$\leq 72$ hrs & $4(40.0)$ & $2(50.0)$ & $6(42.9)$ \\
\hline$>72$ hrs & $1(10.0)$ & $1(25.0)$ & $2(14.3)$ \\
\hline
\end{tabular}

aeruginosa analysis set APACHE, Acute Physiology and Chronic Health Evaluation; CPIS, Clinical Pulmonary Infection Score; SOFA, Sequential Organ Failure Assessment

However, a number of studies suggest that shorter courses of antibiotic therapy for VAP may potentially be less favorable in some circumstances, especially for treatment of infections attributed to NLFGNB. Chastre et al. showed that among patients with VAP, all of whom received appropriate initial empiric antibiotic therapy, comparable clinical effectiveness and outcomes were obtained with 8- and 15-day treatment regimens [5]. Yet, patients with VAP caused by NLFGNB, including $P$. aeruginosa, receiving 8 days of treatment had a higher pulmonary infection recurrence rate compared with those receiving 15 days of treatment (40.6\% versus 


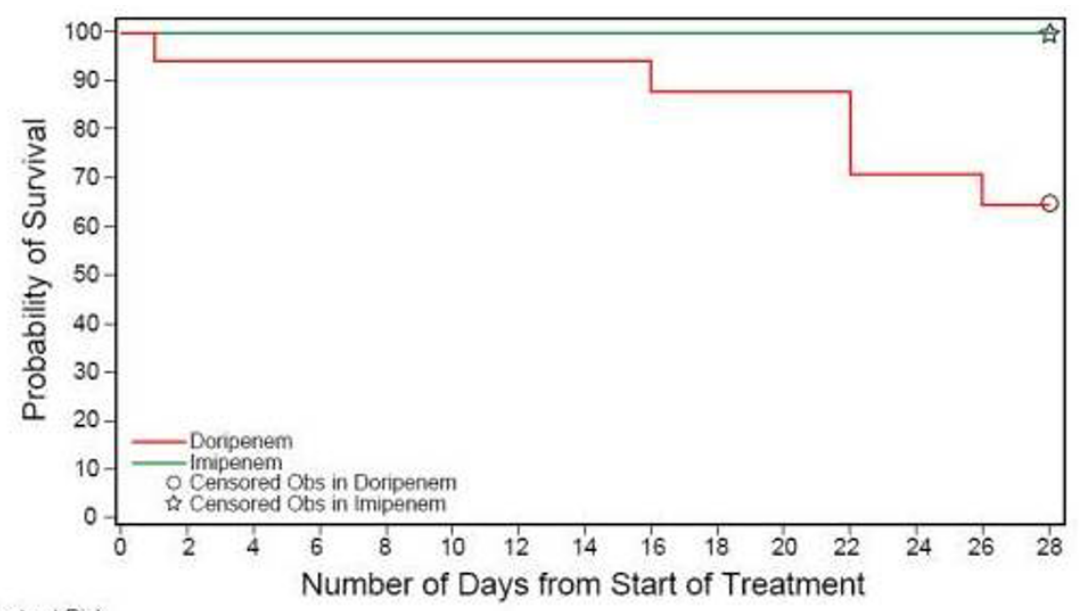

No. Subjects at Risk

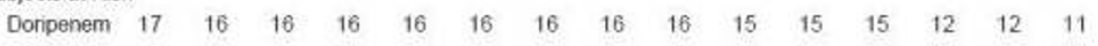

Figure 4 Kaplan-Meier curves for the $\boldsymbol{P}$. aeruginosa subgroup. ( $P=0.040$, Log-Rank Test).

$25.4 \%$; $95 \% \mathrm{CI}, 3.9 \%$ to $26.6 \%)$. Hedrick et al. retrospectively evaluated 154 patients with VAP attributed to NLFGNB where 27 patients were treated with three to eight days (mean $6.4+0.3$ days) of antibiotics and 127 received nine or more days (mean $17.1+0.7$ days) of therapy [19]. Although not statistically different, the mortality rate was higher for patients receiving the shorter treatment courses $(22 \%$ versus $14 \%$; $P=0.38)$. Other investigators have demonstrated that longer courses of antibiotic therapy (10 to 14 days) are typically needed to successfully treat VAP attributed to MDR Gram-negative bacteria, often due to the presence of initial inappropriate antibiotic treatment $[20,21]$.

Several potential explanations may have accounted for our findings. The importance of adequate antimicrobial dosing as a determinant of outcome has been demonstrated in several randomized controlled trials performed in critically ill patients with nosocomial pneumonia [22-24]. Potentially, inadequately dosed antibiotics (ceftobiprole and tigecycline) were associated with statistically greater treatment failures and mortality compared to more optimally dosed comparators. Additionally, the results from two recent meta-analyses examining prolonged infusion of $\beta$-lactam antibiotics found that continuous infusion of $\beta$-lactam antibiotics led to the same clinical results as similar or higher dosed intermittent infusion antibiotic therapy $[25,26]$. However, in one of these meta-analyses, a trend towards benefit among patients receiving intermittent infusion antibiotics possibly explained by the use of higher antibiotic doses was observed [26]. The findings from a recent study examining epithelial lining fluid (ELF) concentrations of doripenem in normal volunteers reported the area under the curve ELF to plasma ratios was comparable to other carbapenems and supports further the use of the $1 \mathrm{~g}$ dose versus a $500 \mathrm{mg}$ dose administered as a four-hour infusion to achieve higher doripenem levels in the ELF [27].

A population $\mathrm{PK}$ analysis to characterize the $\mathrm{PK}$ of doripenem in patients with VAP using data from subjects treated with doripenem from this study and patients with VAP from previous studies showed good PK coverage above MICs in this study, including the subjects with supranormal creatinine clearance. In addition, the population $\mathrm{PK} / \mathrm{PD}$ analysis demonstrated no association between clinical outcomes and the infecting pathogen MICs within the PK dataset from this study. Plasma levels of doripenem were collected between study Days 2 and 3 so the ability to determine these doripenem exposure-response relationships closer to the time that the study endpoint was assessed was limited. However, notably, for subjects in both treatment arms, cure rates and mortality did not appear to increase with increasing MIC of the pathogens, suggesting conditions other than MIC and antibiotic dosing played a role in outcome. Furthermore, the mean distribution of CPIS values during antibiotic treatment for the first seven days of the study was similar for the two treatment groups (Figure 3), only separating after completion of therapy in the doripenem arm. This finding also suggests that the duration of antibiotic therapy and not the antibiotics themselves contributed to the observed differences in outcomes.

Another potential explanation for our findings is that achievement of the targeted antibiotic concentration goals took longer with the use of the prolonged infusion 
compared to the shorter infusions of the time-dependent carbapenems $[28,29]$. This could result in a delay in clearance of the infection that could adversely influence outcomes. The authors of a recent systematic review describing the pharmacokinetics of $\beta$-lactam antibiotics in the critically ill found that $\beta$-lactam antibiotic half-life and time above the MIC were virtually unpredictable, especially in those with normal renal function [30]. Moreover, two recent studies found that creatinine clearance appears to be an important predictor of sub-therapeutic $\beta$-lactam concentrations in critically ill patients [31,32]. Administration of a loading dose of doripenem might have improved concentration target attainment potentially allowing for a shorter course of effective therapy, even in the setting of increased drug clearance $[30,32]$.

This study has several important limitations. First, clinical outcomes were assessed within 24 hours after administration of the last dose of blinded study drug. The timing of this visit provided three days for patients in the doripenem arm but less than 24 hours in the imipenemcilastatin arm to relapse. Had the clinical outcome assessment been postponed to a few days later, additional relapses may have been observed in the imipenem-cilastatin arm. Second, premature closure of the study limited the number of patients in the MITT group and pathogen-specific subgroups. Third, there were a large number of study sites located in many countries which likely have different treatment practices, introducing additional variability. Fourth, enrollment occurred over the course of three years and we cannot exclude temporal changes in supportive care or other practices also having introduced additional variability. Fifth, allowing pathogens with MICs greater than $8 \mu \mathrm{g} / \mathrm{mL}$ may have influenced our results, especially for the shorter course of therapy. Sixth, there were numerically more cases of VAP attributed to $P$. aeruginosa, A. baumannii and MRSA in the doripenem arm compared to the imipenem-cilastatin arm and other imbalances in baseline characteristics between treatment groups which may have contributed to the study findings. Lastly, there may be additional imbalances between the two study groups that could have increased the severity of VAP (for example, corticosteroid therapy, chronic obstructive pulmonary disease) that were not included in our analysis.

\section{Conclusions}

In summary, we demonstrated that a fixed 7-day course of doripenem was found to have non-significant higher rates of clinical failure and mortality compared to a fixed 10-day course of imipenem-cilastatin and a statistically greater mortality for the subgroup of VAP attributed to $P$. aeruginosa. Given the increasing prevalence of VAP caused by MDR Gram-negative bacteria, it is imperative that optimal antimicrobial treatment strategies be employed to optimize efficacy while minimizing the emergence of further antibiotic resistance [33]. In countries where doripenem $500 \mathrm{mg}$ one-hour and four-hour infusions are approved to treat adults with nosocomial pneumonia, including VAP, the usual treatment duration is 7 to 14 days and should be guided by the severity of illness, infecting pathogen and the patients' clinical response with consideration given to treating patients with VAP for more than 7 days to optimize clinical outcome. Moreover, the European Medicines Agency (EMA) has recently recommended that doripenem $1 \mathrm{~g}$ doses administered every eight hours as four-hour infusions be considered in patients with augmented renal clearance (particularly those with creatinine clearance $\geq 150 \mathrm{ml} /$ min) and/or in infections due to non-fermenting Gramnegative pathogens, such as $P$. aeruginosa and Acinetobacter spp. Moreover, the EMA highlighted that the usual treatment duration for patients with nosocomial pneumonia, including VAP, is 10 to 14 days and often in the upper range for patients infected with NLFGNB [34].

\section{Key messages}

- A fixed 7-day course of doripenem was found to have non-significant higher rates of clinical failure and mortality compared to a fixed 10-day course of imipenem-cilastatin for the treatment of VAP.

- VAP due to NLFGNB should be treated with antibiotic courses that are longer than seven days.

- The use of prolonged infusion antibiotic therapy for VAP needs additional study to determine its relative efficacy compared to standard therapy.

\section{Additional material}

Additional file 1: A randomized trial of 7-day doripenem versus 10-
day imipenem-cilastatin for ventilator-associated pneumonia on-
line supplement. Provides additional methods section, results and the
IDMC charter.

\section{Abbreviations}

AE: adverse event; APACHE: Acute Physiology and Chronic Health Evaluation; BAL: bronchoalveolar lavage; Cl: confidence interval; CPIS:clinical pulmonary infection score; ELF: epithelial lining fluid; EMA: European Medicines Agency; EOT: end of therapy; ESBL: extended-spectrum beta-lactamase; $\mathrm{FiO}_{2}$ : fraction of inspired oxygen; GCP: good clinical practices; ICU: intensive care unit; IDMC: Independent Data Monitoring Committee; ITT: intent-to-treat; MDR: multidrug-resistant; MIC: minimum inhibitory concentration; MITT: microbiological intent-to-treat; MRSA: methicillin-resistant Staphylococcus aureus; NLFGNB: non-lactose fermenting Gram-negative bacteria; $\mathrm{PaO}_{2}$ : partial pressure of arterial oxygen; PD: pharmacodynamic; PK: pharmacokinetic; VAP: ventilator-associated pneumonia

\section{Acknowledgements}

Independent Data Monitoring Safety Committee consists of Heidi M. Jolson, MD, MPH, Chevy Chase, Maryland; Marin H. Kollef, MD, Washington University School of Medicine, St. Louis, Missouri; Marcos Restrepo, MD, 
University of Texas Health Science Center at San Antonio, South Texas Veterans Health Care System; and Iza Peszek, PhD, Independent Professional Services, Lafayette, CO.

Study investigators who enrolled at least one subject (the study protocol was approved by the Institutional Review board from each of the participating study sites) are as follows: Aguirre, Guadalupe, M.D. (Hospital Civil de Guadalajara, Guadalajara Jalisco, Mexico); Albrecht, Roxie Mae, M.D. (University of Oklahoma Health Sciences Center, Oklahoma City, OK 73104, USA); Alp Mese, Emine, M.D., Prof, Dr. (Erciyes UMF, Department of Clinical Bacteriology and Infectious Diseases, Kayseri, Turkey); Ambasch, Germán, M. D. (Hospital San Roque, Córdoba (5000), Argentina); Balik, Ismail, M.D. (Ankara UMF, Department of Clinical Bacteriology and Infectious Diseases, SıhhıyeAnkara-, Turkey); Bochicchio, Grant, M.D. (University of Maryland Medical Center, R. Adams Cowley Shock Trauma Center, Baltimore, MD 21201, USA); Bychkova, Larisa V., M.D., Ph.D. (State Public Health Organization of Moscow, Moscow 119415, Russia); Cavalcanti, Manuela, M.D. (Santa Casa de Misericórdia de Porto Alegre, Brazil); Chapman, Marianne, MBBS, Ph.D. (Royal Adelaide Hospital, Adelaide SA 5000, Australia); Clavel, Marc, M.D. (Centre Hospitalier Universitaire de Limoges, 87042 Limoges Cedex, France); DePietro, Michael, M.D. (Christiana Hospital, Newark, DE 19718 USA); Ershova, Olga B., M.D., Ph.D., Doctor of Medical Sciences, Prof. (Municipal Organization of Public Health "Clinical Hospital of Emergency Care n.a. N.V. Solovyev", Yaroslavl 150003, Russia); Fedorova, Tatiana A., M.D., Ph.D., Doctor of Medical Sciences, Prof. (State Public Health Organization of Moscow "City Clinical Hospital n.a. S. P. Botkin", Moscow 125284, Russia); Fejér, Csaba. M.D. (Fővárosi Önkormányzat Szent Imre Kórháza, Sürgősségi Betegellátás Centrum, 115 Budapest, Hungary); Freire, Antônio Tarcísio, M.D. (Santa Casa de Misericórdia de Belo Horizonte, Brazil); Garber, Gary, M.D., FRCPC (The Ottawa Hospital-General Campus, Ottawa, Ontario, K1H 8L6, Canada); Garot, Denis, M.D. (CHU Bretonneau, Réanimation Médicale Polyvalente, 37044 Tours Cedex 9, France); Glumcher, Felix, M.D., Ph.D. (National Medical University, 01133 Kiev, Ukraine); Gonzalez, Magdalena, M.D. (Hospital Nacional de Cuilapa, Cuilapa Santa Rosa, Guatemala); Jauregui, Arturo, M.D. (Hospital CIMA, Chihuahua, Chihuahua, Mexico); Kerwin, Andrew, M.D. (University of Florida/Shands Medical Center, Jacksonville, FL 32209, USA); Köksal, İftihar, M.D., Prof, Dr. (Karadeniz Technical UMF, Department of Clinical Bacteriology and Infectious Diseases, Trabzon, Turkey); Kujath, Peter, Prof. Dr. (Universitätsklinikum Schleswig-Holstein, Lübeck, Germany); Leblebicioglu, Hakan, M.D., Prof., Dr. (Department of Clinical Bacteriology and Infectious Diseases -Kurupelit Samsun, Turkey); Lima, Maria Patelli, M.D. (Hospital e Maternidade Celso Pierro, Brazil); Lobo, Suzana Margarete Ajeje, M.D. (Fundação Faculdade Regional de Medicina de São José do Rio Preto, Brazil); Lopez, Diego, M.D. (Hospital Fundación Jiménez Díaz, 28040 Madrid, Spain); Lorx, András, M.D., Ph.D (Semmelweis Egyetem, Anaesthesiológiai és Intenzív Terápiás Klinika, H-1125 Budapest, Hungary); Maclsaac, Christopher. MBBS, Ph.D. (Royal Melbourne Hospital, Parkville, VIC 3050, Australia); Magyar, Prof. Pál M.D., Ph.D. (Semmelweis Egyetem, Pulmonológiai Klinika, H-1125 Budapest, Hungary); Mangrulkar, Sanjeev, M.D. (Deenanath Mangeshkar Hospital, Erandawana, Pune 411004, India); Mañez Rafael, M.D., Ph.D. (Hospital de Bellvitge, 08907 (Barcelona). Spain); Moralejo, Juan Pablo, M.D. (Hospital Roosevelt, Zona 11, Guatemala Guatemala); Nikonov, Vadim, M.D., Ph.D. (Kharkiv Medical Academy of Postgraduate Education, 61018 Kharkiv, Ukraine); Nyulasi, Tibor M.D. (Fővárosi Önkormányzat Szent János Kórháza, Központi Intenzív és Anaesthesiológia, H-1125 Budapest, Hungary); Papazian, Laurent, M.D. (Centre Hospitalier Universitaire - Hôpital Nord, Réanimation Détresses Respiratoires et Infections Sévères, 13915 Marseille Cedex 20, France); Pareigis, Alex, M.D., (Moline, IL, USA); Plantefeve, Gaëtan, M.D. (CH Victor Dupouy d'Argenteuil, Service de Réanimation Polyvalente, 95107 Argenteuil, France); Poirier, Germain, M.D., C.M., FRCPC (Québec,J4V 2H1, Canada); Ragaller, Maximilian, M.D. (Medizinische Klinik und Poliklinik 1, MK1A1, Dresden, Germany); Rahav, Galia, Prof, M.D. (Sheba Medical Center, Ramat-Gan 52622, Israel); Ray, Sumit M.D. (Sir Ganga Ram Hospital, New Delhi 110060, India); Rendon, Adrian (Hospital Universitario de Monterrey, Monterrey Nuevo Leon, Mexico); Rodríguez, Alejandro Hugo, M.D., Ph.D. (Hospital Universitari Joan XXIII, 43007 Tarragona, Spain); Rozgonyi, Zsolt, M. D. (Fővárosi Önkormányzat Péterfy Sándor utcai Kórház-Rendelőintézet és Baleseti Központ, H-1081 Budapest, Hungary); Sánchez Senen, Alejandro, M.D. (Policlínico Modelo de Cipolletti, Cipolletti, Rio Negro, Argentina); Sandesc, Dorel, M.D. (Spitalul Clinic Judetean de Urgenta Nr 1, Timisoara, Jud. Timis, Romania); Sarkany, Ágnes M.D. (Fejér Megyei Szent György Kórház, Központi, H-8000 Székesfehérvár, Hungary); Shlapak, Igor, M.D., Ph.D. (National Medical
Academy for Postgraduate Education, 02660 Kiev, Ukraine); Simanenkov, Vladimir I., M.D., Ph.D., Doctor of Medical Sciences, Prof. (Saint-Petersburg State Public Health Organization "City Clinical Hospital \#26" Saint-Petersburg 196247, Russia); Smolyanyy, Oleksander, M.D., Ph.D. (Odessa Regional Clinical Hospital, 65025 Odessa, Ukraine); Solé-Violen, Jorge, M.D. (Hospital Universitario de Gran Canaria Doctor Negrin, 35020 Las Palmas de Gran Canaria, Spain); Stuttmann, Ralph, M.D. (Halle/Saale, Germany); Vazquez, Jose, M.D. (Henry Ford Hospital, Detroit, MI 48202,USA); Walker, Craig, MBBS (Monash Medical Centre, Clayton, VIC 3168, Australia); Wiwatworapan, Tawatchai, (Maharat Nakhonratchasima Hospital, Nakhonratchasima 30000, Thailand); Zelaya, Ivonne, M.D. (Hospital Nacional de Escuintla, Carretera a Taxisco, Escuintla, Guatemala) We thank Kim Taylor for outstanding efforts in supervising the operational activities of this study, and to Partha Nandy, Partha Bagchi PhD and Paul Kotey PhD for help in pharmacometrics and statistical analyses.

\section{Author details}

${ }^{1}$ Division of Pulmonary and Critical Care Medicine, Washington University School of Medicine, St. Louis, 660 South Euclid Avenue, Campus Box 8052, St. Louis, MO 63110, USA. ²Service de Réanimation Médicale, Institut de Cardiologie, Groupe Hospitalier Pitié-Salpêtrière, 47-83 boulevard de l'Hôpital, 75651 Paris Cedex 13, France. ${ }^{3}$ Centre Hospitalier De Limoges, Hospital Dupuytren 02 Ave, Martin Luther King 87000, Limoges, France. ${ }^{4}$ Division of Pulmonary/Critical Care Medicine, University of Texas Health Science Center at San Antonio and Division Pulmonary/Critical Care Medicine, South Texas Veterans Health Care System, 7400 Merton Minter Blvd. (11c6), San Antonio, TX, 78229 USA. 5Janssen Research and Development, Turnhoutsewig 30,

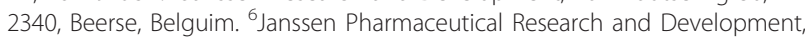
1000 Route 202, Raritan, NJ, 08869 USA.

\section{Authors' contributions}

MK, JC, MC, MR, MB, KK and RR had full access to all of the data in the study and take responsibility for the integrity of the data and the accuracy of the data analysis. MK, JC, MC, MR, MB, KK, RR, IC and HK contributed to the study conception and design, statistical analysis, drafting of the manuscript, and have given approval to the final version. IC and HK were the sponsor's designated clinical pharmacologist and modeling scientist responsible for pharmacokinetic analysis of the data and critical revision of the manuscript.

\section{Competing interests}

This study was funded by Janssen Pharmaceutical Research and Development. Dr. Kollef's effort was supported by the Barnes-Jewish Hospital Foundation and Dr. Kollef has received consulting fees from Janssen. Dr. Restrepo's time is partially protected by Award Number K23HL096054 from the National Heart, Lung, and Blood Institute. The content of this manuscript is solely the responsibility of the authors and does not necessarily represent the official views of the National Heart, Lung, and Blood Institute or the National Institutes of Health." The funding agencies had no role in the preparation, review or approval of the manuscript. The views expressed in this article are those of the authors and do not necessarily represent the views of the Department of Veterans Affairs, nor the University of Texas Health Science Center at San Antonio. The remaining authors have no competing interests to declare.

Received: 5 September 2012 Revised: 28 September 2012 Accepted: 9 November 2012 Published: 13 November 2012

\section{References}

1. Kollef $M H$ : Ventilator-associated pneumonia. A multivariate analysis. JAMA 1993, 270:1965-1970.

2. Vincent JL, Rello J, Marshall J, Silva E, Anzueto A, Martin CD, Moreno R, Lipman J, Gomersall C, Sakr Y, Reinhart K, EPIC II Group of Investigators: International study of the prevalence and outcomes of infection in intensive care units. JAMA 2009, 302:2323-2329.

3. American Thoracic Society Infectious Diseases Society of America: Guidelines for the management of adults with hospital-acquired, ventilator-associated, and healthcare-associated pneumonia. Am J Respir Crit Care Med 2005, 171:388-416.

4. Rotstein C, Evans G, Born A, Grossman R, Light RB, Magder S, McTaggart B, Weiss K, Zhanel GG: Clinical practice guidelines for hospital-acquired 
pneumonia and ventilator-associated pneumonia in adults. Can J Infect Dis Med Microbiol 2008, 19:19-53.

5. Chastre J, Wolff M, Fagon JY, Chevret S, Thomas F, Wermert D, Clementi E, Gonzalez J, Jusserand D, Asfar P, Perrin D, Fieux F, Aubas S, PneumA Trial Group: Comparison of 8 vs 15 days of antibiotic therapy for ventilatorassociated, pneumonia in adults: a randomized trial. JAMA 2003, 290:2588-2598.

6. Pugh R, Grant C, Cooke RP, Dempsey G: Short-course versus prolongedcourse antibiotic therapy for hospital-acquired pneumonia in critically ill adults. Cochrane Database Syst Rev 2011, 10:CD007577.

7. Fink MP, Snydman DR, Niederman MS, Leeper KV Jr, Johnson RH, Heard SO, Wunderink RG, Caldwell JW, Schentag JJ, Siami GA: Treatment of severe pneumonia in hospitalized patients: results of a multicenter, randomized, double-blind trial comparing intravenous ciprofloxacin with imipenem-cilastatin. Antimicrob Agents Chemother 1994, 38:547-557.

8. Réa-Neto A, Niederman M, Lobo SM, Schroeder E, Lee M, Kaniga K, Ketter N, Prokocimer P, Friedland I: Efficacy and safety of doripenem versus piperacillin/tazobactam in nosocomial pneumonia: a randomized, open-label, multicenter study. Curr Med Res Opin 2008, 24:2113-2126.

9. Chastre J, Wunderink R, Prokocimer P, Lee M, Kaniga K, Friedland I: Efficacy and safety of intravenous infusion of doripenem versus imipenem in ventilator-associated pneumonia: a multicenter, randomized study. Crit Care Med 2008, 36:1089-1096.

10. Van Wart SA, Andes DR, Ambrose PG, Bhavnani SM: Pharmacokineticpharmacodynamic modeling to support doripenem dose regimen optimization for critically ill patients. Diagn Microbiol Infect Dis 2009, 63:409-414.

11. Vaccaro N, Umeh O, Redman R, Cirillo I: Pharmacokinetics of doripenem 1 $\mathrm{g}$ administered over 4 hours in patients with ventilator-associated pneumonia. Proceedings of the 49th Interscience Conference on Antimicrobial Agents and Chemotherapy (ICAAC) San Francisco, California; 2009, 12-15, [Abstract: A1-018].

12. Drusano GL, Lodise TP, Melnick D, Liu W, Oliver A, Mena A, VanScoy B, Louie A: Meropenem penetration into epithelial lining fluid in mice and humans and delineation of exposure targets. Antimicrob Agents Chemother 2011, 55:3406-3412.

13. Crandon JL, Ariano RE, Zelenitsky SA, Nicasio AM, Kuti JL, Nicolau DP: Optimization of meropenem dosage in the critically ill population based on renal function. Intensive Care Med 2011, 37:632-638.

14. Ibrahim EH, Ward S, Sherman G, Schaiff R, Fraser VJ, Kollef MH: Experience with a clinical guideline for the treatment of ventilator-associated pneumonia. Crit Care Med 2001, 29:1109-1115

15. Micek ST, Ward S, Fraser VJ, Kollef MH: A randomized controlled trial of an antibiotic discontinuation policy for clinically suspected ventilatorassociated pneumonia. Chest 2004, 125:1791-1799.

16. Singh N, Rogers P, Atwood CW, Wagener MM, Yu VL: Short-course empiric antibiotic therapy for patients with pulmonary infiltrates in the intensive care unit. A proposed solution for indiscriminate antibiotic prescription. Am J Respir Crit Care Med 2000, 162:505-511.

17. Bouadma L, Luyt CE, Tubach F, Cracco C, Alvarez A, Schwebel C, Schortgen F, Lasocki S, Veber B, Dehoux M, Bernard M, Pasquet B, Régnier B, Brun-Buisson C, Chastre J, Wolff M, PRORATA trial group: Use of procalcitonin to reduce patients' exposure to antibiotics in intensive care units (PRORATA trial): a multicentre randomised controlled trial. Lancet 2010, 375:463-474.

18. Stolz D, Smyrnios N, Eggimann P, Pargger $H$, Thakkar N, Siegemund M, Marsch S, Azzola A, Rakic J, Mueller B, Tamm M: Procalcitonin for reduced antibiotic exposure in ventilator-associated pneumonia: a randomised study. Eur Respir J 2009, 34:1364-1375.

19. Hedrick TL, McElearney ST, Smith RL, Evans HL, Pruett TL, Sawyer RG: Duration of antibiotic therapy for ventilator-associated pneumonia caused by non-fermentative gram-negative bacilli. Surg Infect 2007, 8:589-597.

20. Kollef KE, Schramm GE, Wills AR, Reichley RM, Micek ST, Kollef MH: Predictors of 30-day mortality and hospital costs in patients with ventilator-associated pneumonia attributed to potentially antibioticresistant gram-negative bacteria. Chest 2008, 134:281-287.

21. Florescu DF, Qiu F, McCartan MA, Mindru C, Fey PD, Kalil AC: What is the efficacy and safety of colistin for the treatment of ventilator-associated pneumonia? A systematic review and meta-regression. Clin Infect Dis 2012, 54:670-680.
22. Freire AT, Melnyk V, Kim MJ, Datsenko O, Dzyublik O, Glumcher F, Chuang YC, Maroko RT, Dukart G, Cooper CA, Korth-Bradley JM, Dartois N, Gandjini H, 311 Study Group: Comparison of tigecycline with imipenem/ cilastatin for the treatment of hospital-acquired pneumonia. Diagn Microbiol Infect Dis 2010, 68:140-151.

23. Neuner E, Ritchie D, Micek S: New antibiotics for healthcare associated pneumonia. Semin Respir Crit Care Med 2009, 30:92-101.

24. Basilea announces positive top-line data from phase III study of ceftobiprole in hospital-acquired pneumonia. [http://www.basilea.com/ News-and-Media/Basilea-Announces-Positive-Top-line-Data-from-Phase-IIIStudy-of-Ceftobiprole-in-Hospital-Acquired-Pneumonia/144], [press release] October 9, 2007. Accessed October 10, 2011, at.

25. Tamma PD, Putcha N, Suh YD, Van Arendonk KJ, Rinke ML: Does prolonged $\beta$-lactam infusions improve clinical outcomes compared to intermittent infusions? A meta-analysis and systematic review of randomized, controlled trials. BMC Infect Dis 2011, 11:181.

26. Roberts J, Webb S, Paterson D, Ho K, Lipman J: A systematic review on clinical benefits of continuous administration of beta-lactam antibiotics. Crit Care Med 2009, 37:2071-2078.

27. Justo J, Gotfried MH, Deyo K, Fischer P, Danziger LH, Rodvold KA: Doripenem intrapulmonary pharmacokinetics in healthy adult subjects. Proceedings of the 51st Interscience Conference on Antimicrobial Agents and Chemotherapy, 17-20 September 2011, Chicago, Illinois, [Abstract: A1-1748].

28. Roberts JA, Kirkpatrick CM, Roberts MS, Robertson TA, Dalley AJ, Lipman J: Meropenem dosing in critically ill patients with sepsis and without renal dysfunction: intermittent bolus versus continuous administration? Monte Carlo dosing simulations and subcutaneous tissue distribution. J Antimicrob Chemother 2009, 64:142-150.

29. Bulitta JB, Landersdorfer CB, Forrest A, Brown SV, Neely MN, Tsuji BT, Louie A: Relevance of pharmacokinetic and pharmacodynamic modelling to clinical care of critically ill patients. Curr Pharm Biotechnol 2011, 12:2044-2061.

30. Goncalves-Pereira J, Povoa P: Antibiotics in critically ill patients- a systematic review of the pharmacokinetics of beta-lactams. Crit Care 2011, 15:R206.

31. Udy A, Roberts J, Lipman J: Implications of augmented renal clearance in critically ill patients. Nat Rev Nephrol 2011, 7:539-543.

32. Udy AA, Varghese JM, Altukroni M, Briscoe S, McWhinney B, Ungerer J, Lipman J, Roberts JA: Subtherapeutc initial $\beta$-lactam concentrations in select critically ill patients. Association between augmented renal clearance and low trough drug concentrations. Chest 2012, 142:30-39.

33. Chung DR, Song JH, Kim SH, Thamlikitkul V, Huang SG, Wang H, So TM, Yasin RM, Hsueh PR, Carlos CC, Hsu LY, Buntaran L, Lalitha MK, Kim MJ, Choi JY, Kim SI, Ko KS, Kang Cl, Peck KR, Asian Network for Surveillance of Resistant Pathogens Study Group: High prevalence of multidrug-resistant nonfermenters in hospital-acquired pneumonia in Asia. Am J Respir Crit Care Med 2011, 184:1409-1417.

34. European Medicines Agency: Science Medicines Health:[http://www.ema. europa.eu/ema/index.jsp?curl=pages/medicines/human/ public_health_alerts/2012/06/human_pha_detail_000064. jsp\&mid=\&source=homeMedSearch\&category=human].

doi:10.1186/cc11862

Cite this article as: Kollef et al:: A randomized trial of 7-day doripenem versus 10-day imipenem-cilastatin for ventilator-associated pneumonia. Critical Care 2012 16:R218. 